\title{
Ex vivo localization and immunohistochemical detection of sentinel lymph node micrometastasis in patients with colorectal cancer can upgrade tumor staging
}

Fu-Long Wang ${ }^{\dagger}$, Fang Shen ${ }^{\dagger}$, De-Sen Wan, Zhen-Hai Lu, Li-Ren Li, Gong Chen, Xiao-Jun Wu, Pei-Rong Ding, Ling-Heng Kong and Zhi-Zhong Pan ${ }^{*}$

\begin{abstract}
Background: It is not clear if sentinel lymph node (SLN) mapping can improve outcomes in patients with colorectal cancers. The purpose of this study was to determine the prognostic values of ex vivo sentinel lymph node (SLN) mapping and immunohistochemical (IHC) detection of SLN micrometastasis in colorectal cancers.

Methods: Colorectal cancer specimens were obtained during radical resections and the SLN was identified by injecting a 1\% isosulfan blue solution submucosally and circumferentially around the tumor within 30 min after surgery. The first node to stain blue was defined as the SLN. SLNs negative by hematoxylin and eosin (HE) staining were further examined for micrometastasis using cytokeratin $\mathrm{IHC}$.

Results: A total of 54 patients between 25 and 82 years of age were enrolled, including 32 males and 22 females. More than $70 \%$ of patients were T3 or above, about $86 \%$ of patients were stage II or III, and approximately $90 \%$ of patients had lesions grade II or above. Sentinel lymph nodes were detected in all 54 patients. There were 32 patients in whom no lymph node micrometastasis were detected by HE staining and 22 patients with positive lymph nodes micrometastasis detected by HE staining in non-SLNs. In contrast only 7 SLNs stained positive with HE. Using HE examination as the standard, the sensitivity, non-detection rate, and accuracy rate of SLN micrometastasis detection were 31.8\% (7/22), 68.2\% (15/22), and 72.2\%, respectively. Micrometastasis were identified by $\mathrm{ICH}$ in 4 of the 32 patients with HE-negative stained lymph nodes, resulting in an upstaging rate 12.5\% (4/32). The 4 patients who were upstaged consisted of 2 stage I patients and 2 stage II patients who were upstaged to stage III. Those without lymph node metastasis by HE staining who were upstaged by IHC detection of micrometastasis had a significantly poorer disease-free survival $(p=0.001)$ and overall survival $(p=0.004)$.

Conclusion: Ex vivo localization and immunohistochemical detection of sentinel lymph node micrometastasis in patients with colorectal cancer can upgrade tumor staging, and may become a factor affecting prognosis and guiding treatment.
\end{abstract}

Virtual slides: The virtual slide(s) for this article can be found here: http://www.diagnosticpathology.diagnomx.eu/ vs/1350200526694475.

Keywords: Colorectal carcinoma, Sentinel lymph node, Micrometastasis, Prognosis

\footnotetext{
*Correspondence: panzhzh@sysucc.org.cn

${ }^{\dagger}$ Equal contributors

State Key Laboratory of Oncology in South China; Department of Colorectal

Surgery, Cancer Center, Sun Yat-sen University, 651 Dongfengdong Road,

Guangzhou, Guangdong 510060, P. R. China
} 


\section{Introduction}

Colorectal cancer is the most common malignancy in men and women, and the fourth leading cause of cancer-related death worldwide [1]. The status of lymph nodes in colorectal carcinoma is the most important prognostic factor for recurrence and overall survival (OS), and also largely determines whether adjuvant chemotherapy should be given [2]. Approximately 70-90\% of patients with no lymph node involvement will survive 5 years, whereas only $40 \%$ of those with lymph node metastases will survive for that length of time. However, about $30 \%$ of patients with node-negative colon cancer staged by standard pathologic techniques experience recurrent locoregional or distant metastases within 5 years $[1,2]$. One of the possible reasons for the variations in outcome among patients with node-negative disease is that there is inaccurate or incomplete nodal staging [3].

All lymph nodes within resected specimens should be examined after surgery to obtain an accurate N stage; however, small lymph nodes with metastases are likely to be missed during conventional gross pathological examination. Study has shown that $>70 \%$ of the positive lymph nodes are $<2 \mathrm{~mm}$ [4]. The benchmark of 12 as a minimum acceptable number of lymph nodes obtained during colorectal cancer resection has been adopted as a quality measure for surgical practice by many organizations. Even so, two large population-based studies found that only $37-$ $49 \%$ of patients with colon cancer receive an adequate lymph node evaluation [5-7]. Techniques such as fat clearance are used to increase the yield of lymph nodes, but these methods are time-consuming and not used routinely.

Several studies have reported nodal micrometastasis in colorectal cancer by examination methods such as serial sectioning, immunohistochemistry (IHC), or reverse transcriptase-polymerase chain reaction (RT-PCR). However, it is not clear if these findings indicate a worse prognosis [5,8-11]. In addition, these ultrastaging techniques are too costly and time-consuming for use on the 12 or more lymph nodes in every colorectal cancer specimen.

The sentinel node (SLN) is the first lymph node to receive lymphatic drainage from a tumor. In concept, the SLN has the highest chance of harboring tumor cells because they directly drain from the tumor. The concept of SLN allows focused examination on just a few nodes at greatest risk for metastatic involvement. If lymph node staging can be upgraded by focusing on detection of a few SLNs using conventional and immunohistochemical techniques, better clinical outcomes may be expected. With successful application of SLN detection technique in breast cancer [12-14], investigators have attempted to apply this technique in colorectal cancer. Many studies have demonstrated the feasibility of applying SLN detection techniques in colorectal cancer; however, the detection rate, sensitivity, and false negative rate vary considerably [1,3,4,15-26].
Moreover, as yet only a few studies have shown that survival of colorectal cancer patients can be influenced by the application of the SLN technique [18,19,27].

The purpose of this study was to determine the prognostic value of ex vivo sentinel lymph node (SLN) mapping and immunohistochemical detection of SLN micrometastasis in colorectal cancers.

\section{Materials and methods \\ Patients}

A total of 54 patients with surgery-naive colorectal cancers who received radical operations at the Department of Abdominal Surgery, Sun Yat-sen University Cancer Center between March and October of 2003 were included in this study. All patients had proven colorectal adenocarcinoma by preoperative pathological biopsy examination, and all received clinical examination, chest X-ray, abdominal ultrasound, colonoscopy, and thoracoabdominal computed tomography (CT) scans. Patients with multiple primary colorectal tumors, tumor local excision, severe local infiltration, surgery in tumor lymphatic drainage areas, emergency operations, non-en bloc resections, and M1 patients preoperatively or intraoperatively were excluded from this study. Informed consent was obtained from all patients, and this study was approved by the Institutional Review Board of our hospital.

\section{SLN mapping}

Enrolled patients received standard radical operations per conventional procedures after routine preoperative preparations. SLN localization was performed on ex vivo specimens within 30 minutes or resection using 1\% isosulfan blue solution (Sigma-Aldrich USA). The specimen was opened at the anti-mesenteric border and 0.5-2 ml (depending on the volume of the tumor) 1\% isosulfan blue solution was injected submucosally and circumferentially around the tumor. The injection sites were then gently massaged for 5 minutes to push the tracer into the lymphatic vessels. The first node to stain blue was defined as the SLN.

\section{Pathologic examination}

The SLNs were excised and processed separately. Thereafter, a minimum of 12 non-SLNs as recommended by the UICC/AJCC guidelines were dissected from the specimen. All lymph nodes were collected by the same surgeon. Nodal samples were fixed in formalin and embedded in paraffin. All lymph nodes were sectioned at least one section at 5 um thickness, and were separated for hematoxylin and eosin (HE) staining. SLNs that were determined negative by conventional pathological methods were further examined using cytokeratin (CK; monoclonal mouse anticytokeratin AE1/AE3; Abcam,1:100) IHC to identify micrometastasis. Sentinel nodes were examined using at least 
four multilevel sections at 250-um intervals. Positive lymph node micrometastasis was defined by identification of one or more CK-labeled positive cells in lymph nodes in which metastases were not detected by HE staining in combination with histological and cell morphological findings. All samples, including tumors, nodes, HE-stained, and IHCstained sections were evaluated separately by two experienced pathologists. If different results were obtained, a third pathologist reviewed the specimens and slides to make the final determination.

SLNs and non-SLNs which were positive for micrometastasis by $\mathrm{HE}$ staining were categorized as $\mathrm{N}+$, negative lymph nodes were categorized as N0, and SLNs with micrometastasis detected by IHC staining only were categorized as $\mathrm{NO}(\mathrm{i}+)$. All treatment decisions were based upon the results of standard HE histopathological staging. Patients with stage II and III disease were given fluorouracil-based adjuvant chemotherapies.

\section{Patient follow-up}

All patients were followed-up through clinic visits. Follow-up included clinical examination, carcinoembryonic antigen (CEA) level, chest X-ray, and abdominal ultrasound every 3-6 months for 2 years, then every 612 months. Patients with multiple polyps in the colon received colonoscopies annually, and those with vessel invasion or poor differentiation also received CT scans annually.

\section{Statistical analysis}

Overall survival (OS) duration was calculated from the date when a patient was enrolled in the study to the date of death or last follow-up visit. Disease-free survival (DFS) duration was defined as the interval between the date of enrollment in the study and the date recurrence or metastasis was identified.

Data were presented as mean \pm standard deviation for continuous variables and number (percentage) for categorical variables. Kaplan-Meier survival curves were calculated, and the difference in survival statuses among the three groups $(\mathrm{N}+, \mathrm{N} 0$, and $\mathrm{NO}(\mathrm{i}+))$ was tested by the Breslow estimation method. A Cox proportional hazard model was used to determine the factors associated with survival status, recurrence, and metastasis. Factors with $\mathrm{p}<0.1$ revealed by univariate Cox proportional hazard model analysis, age, and sex were then included in further multiple analyses in order to establish the final model. The forward stepwise method was applied when establishing the multiple Cox proportional model. Statistical significance was defined as $\mathrm{p}<0.05$. All statistical analyses were performed using PASW software (version 18.0, IBM SPSS Inc., Chicago, IL).

In addition, detection rate, accuracy, sensitivity, and false negative rate were reported for the examination of
SLN localization. These indexes were determined in accordance of following formulas [20]:

Detection rate (\%): (number of patients with successfully retrieved SLNs/number of patients enrolled) $\times 100$.

Sensitivity (\%) of lymph node micrometastasis: (number of patients with positive SLNs/number of patients with micrometastasis in any lymph node) $\times 100$.

False-negative rate (\%): 100 - sensitivity

Accuracy (\%): (number of patients with correct nodal status predicted/number of patients enrolled) $\times 100$.

Lymph node ratio: (number of positive lymph nodes/ total number of lymph nodes $) \times 100$.

\section{Results}

Patients and clinical characteristics

A total of 54 patients between 25 and 82 years of age were enrolled, including 32 males and 22 females. Patient demographic and clinical data are shown in Table 1. More than $80 \%$ of patients were stage T3 or above, about $86 \%$ of patients were stage II or III, and approximately $90 \%$ of patients had lesions grade II or above.

\section{Table 1 Patient demographic and clinical data}

\begin{tabular}{lc}
\hline Variables & \multicolumn{1}{c}{ Statistics } \\
\hline Age (y) & $57.00 \pm 13.82$ \\
Lymph node ratio & $0.05 \pm 0.10$ \\
Survival duration (months) & $61.69 \pm 17.20$ \\
DFS duration (month) & $56.33 \pm 23.38$ \\
Gender & \\
Male & $32(59.3)$ \\
Female & $22(40.7)$ \\
Location of tumor & \\
Colon & $28(51.9)$ \\
Rectum & $26(48.1)$ \\
Depth of invasion & \\
T1 & $2(3.7)$ \\
T2 & $8(14.8)$ \\
T3 & $21(38.9)$ \\
T4 & $23(42.6)$ \\
Stage & \\
I & \\
II & $7(13.0)$ \\
II & $25(46.3)$ \\
Reath & $22(40.7)$ \\
\hline DFS, dirrence or metastasis & $13(24.1)$ \\
& $14(25.9)$ \\
\hline
\end{tabular}

DFS, disease free survival; $\mathrm{HE}$, hematoxylin-eosin; SLN, sentinel lymph node. Continuous variables were shown as mean \pm standard deviation and categorical variables were presented as number (\%). 


\section{HE staining for micrometastasis detection}

Sentinel lymph nodes were detected in all 54 patients. There were 32 patients in whom no lymph node micrometastasis were detected by HE staining (N0) and 22 patients with positive lymph nodes micrometastasis detected by $\mathrm{HE}$ staining $(\mathrm{N}+)$ in non-SLNs. In contrast only 7 SLNs stained positive with HE (Table 2). Using HE examination as the standard, the sensitivity, nondetection rate, and accuracy rate of SLN micrometastasis detection were $31.8 \%(7 / 22), 68.2 \%(15 / 22)$, and $72.2 \%$ $(32+7=39 / 54)$, respectively.

Further examination for lymph node micrometastasis by $\mathrm{ICH}$ in patients who were $\mathrm{HE}(-)$ showed that 12 of the 47 patients were positive for micrometastasis in SLNs (Table 3). In those patients, 4 of 12 had non-SLNs that were HE-negative $(\mathrm{NO}(\mathrm{i}+))$, and 8 patients had nonSLNs that were HE-positive. The 4 patients who were upstaged consisted of 2 stage I patients and 2 stage II patients who were upstaged to stage III; thus the upstaging rate was $12.5 \%$ (4/32 of non-SLN $\mathrm{HE}(-)$ patients). Additionally, based on the assumption that the results of micrometastasis detection could be a reference in terms of stage, the accuracy rate was increased from $72.2 \%$ to $87 \%[(7+32+8) / 54]$.

\section{Survival analysis}

Among the 54 patients, 4 patients with micrometastasis detected in SLNs by IHC staining only were upstaged $(\mathrm{NO}(\mathrm{i}+)$, group $\mathrm{A}), 28$ patients had negative $\mathrm{HE}$ and IHC staining (N0, group B), and the other 22 patients had positive $\mathrm{HE}$ staining $(\mathrm{N}+$, group $\mathrm{C})$. In groups $\mathrm{A}$ and $\mathrm{B}$, two patients in each group had metastatic disease and died, and in group C, 10 had recurrent or metastatic disease and 9 died.

The Kaplan-Meier OS curve is shown in Figure 1. The survival status of the three groups were significantly different $(p=0.003)$. Regardless of upstaging status, all patients were alive 1 year after they participated in the study. At the third and the fifth year, the OS of group A was reduced to $75 \%$ and $50 \%$, respectively; that of group $\mathrm{B}$ also declined to $96.3 \%$ and $92.3 \%$, respectively; and that of group C were $90.5 \%$ and $53.5 \%$ respectively.

The Kaplan-Meier DFS curve is shown in Figure 2. In group A, the 1- and 3-year DFS was $100 \%$ and $50 \%$, respectively, and no further change was noted. From 100\% at the first year, the DFS rate of group B decreased to $96.4 \%$ and $92.4 \%$ at 3 - and 5-years, respectively. In group

Table 2 HE examination for micrometastasis in SLNs ( $\mathrm{N}=54)$

\begin{tabular}{lll}
\hline HE staining & Non-SLN & SLN \\
\hline Positive $(\mathrm{N}+)$ & 22 & 7 \\
Negative $(\mathrm{NO})$ & 32 & 47 \\
\hline
\end{tabular}

$\mathrm{HE}$, hematoxylin-eosin; SLN, sentinel lymph node.
Table 3 IHC staining for micrometastasis in patients with HE-negative SLNs $(n=47)$

\begin{tabular}{ll}
\hline ICH staining & SLN \\
\hline Negative & 35 \\
Positive & $12^{*}$ \\
\hline
\end{tabular}

IHC, immunohistochemistry; HE, hematoxylin-eosin; SLN, sentinel lymph node. *Patients with positive ICH staining, of whom 4 of 12 had HE-negative stained lymph nodes and 8 had HE-negative stained SLNs but HE-positive stained non-SLNs at primary HE staining.

C, the 1-year DFS rate was $86.1 \%$, and decreased to $76.0 \%$ and $47.5 \%$ at 3 - and 5-years, respectively. The three groups had statistically different DFS status $(\mathrm{p}=0.001)$.

\section{Cox proportional hazard model}

The results of Cox proportional hazard model with regard to survival are summarized in Table 4. The hazard of death was increased as the lymph node ratio increased (hazard ratio $[\mathrm{HR}]=1.08,95 \%$ confidence interval $[\mathrm{CI}]$ : 1.03-1.13, $\mathrm{p}=0.003)$. Moreover, patients with an upstage of $\mathrm{NO}(\mathrm{i}+)(\mathrm{HR}=10.28,95 \% \mathrm{CI}: 1.44-73.34, \mathrm{p}=0.020)$ and $\mathrm{N}+(\mathrm{HR}=7.93,95 \% \mathrm{CI}:$ 1.71-36.83, $\mathrm{p}=0.008)$ had a higher hazard of death as compared with those with an upstage of N0. In addition, patients with positive HE staining were more likely to be dead ( $\mathrm{HR}=4.82,95 \% \mathrm{CI}$ : 1.48-15.70, $\mathrm{p}=0.009$ ). However, HE staining and lymph node ratio were dropped in the multivariate model; only the effect of upstaging was significant.

Similar results were found for recurrence or/and metastasis (Table 5). In univariate analysis, significant factors were lymph node ratio, upstaging, and positive HE staining; whereas, once again upstage was the only significant

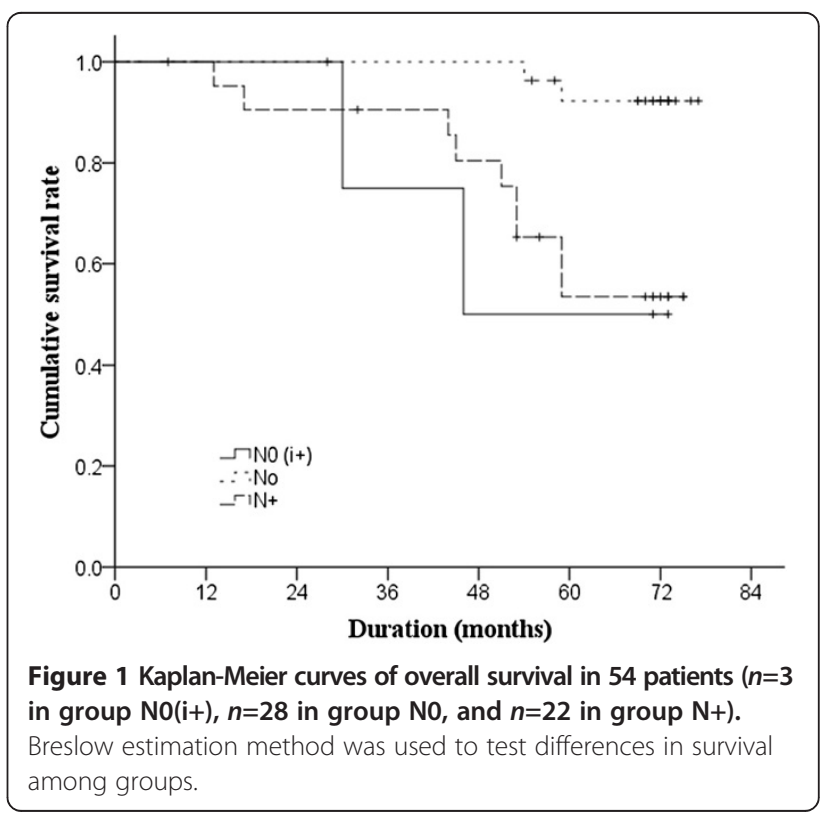




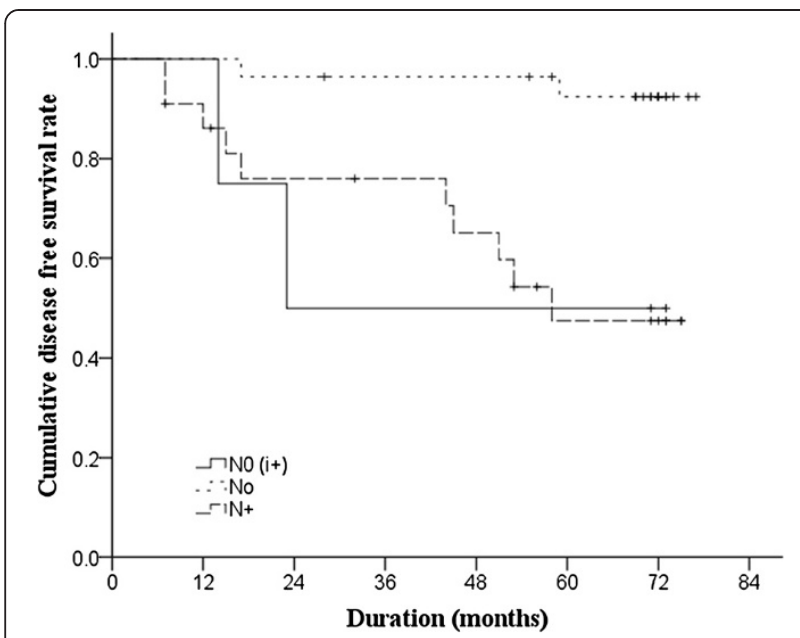

Figure 2 Kaplan-Meier curves of disease-free survival in 54 patients ( $n=3$ in group NO(i+), $n=28$ in group NO, and $n=22$ in group $\mathrm{N}+$ ). Breslow estimation method was used to test differences in disease-free survival among groups.

factor left in the final model. The hazard of death in $\mathrm{NO}(\mathrm{i}+)$ and $\mathrm{N}+$ patients was 8.07 (95\% CI: 1.09-59.50, $\mathrm{p}=0.041$ ) and 7.87 (95\% CI: 1.68-36.79, $\mathrm{p}=0.009)$ times higher than those with N0 disease.

\section{Discussion}

Lymph node metastasis is one of the key factors of poor outcomes in patients with colorectal cancer. However, recurrence and metastasis rates are high in stage II colorectal cancer patients even without lymph node metastasis, and it has been reported that stage IIB patients have a poorer prognosis than stage III patients $[11,21]$. Inaccurate lymph node staging may be one of the reasons for the low survival rate. Methods examined to improve lymph node staging include increasing number of lymph nodes for postoperative examination and using micrometastasis detection; however, it is difficult to apply these techniques in clinical practice due to procedural complexity and costs.

Theoretically, SLN detection can help reduce understaging because the SLN is the first to receive drainage from the tumor. In our study, ex vivo SLN detection was used in 54 patients and we found an identification rate of $100 \%$ and 4 patients were upstaged. Our results are consistent with other studies that have shown SLN mapping results in a higher detection rate and upstaging when combined with immunohistochemical or RT-PCR techniques $[1,16,17,20,22]$. A prospective randomized study of SLN ultrastaging showed that SLNs were successfully identified in 82 of 84 patients (97.6\%), and significant nodal upstaging occurred $(38.7 \%$ to $57.3 \%$, $\mathrm{p}=0.019$ ) [22]. Another prospective multicenter trial demonstrated that at least one SLN was identified in 268 of 315 enrolled patients (detection rate, $85 \%$ ) and 21\%
Table 4 Cox proportional hazard model for survival status

\begin{tabular}{|c|c|c|}
\hline & Univariate & Multivariate \\
\hline Age (y) & $0.99(0.95-1.03)$ & $0.99(0.96-1.04)$ \\
\hline Lymph node ratio $^{\dagger}$ & $1.08(1.03-1.13)^{*}$ & - \\
\hline \multicolumn{3}{|l|}{ Gender } \\
\hline Female & Reference & Reference \\
\hline Male & $2.49(0.69-9.06)$ & $1.16(0.28-4.81)$ \\
\hline \multicolumn{3}{|l|}{ Location of tumor } \\
\hline Colon & Reference & - \\
\hline Rectum & $1.06(0.36-3.16)$ & - \\
\hline \multicolumn{3}{|l|}{ Depth of invasion } \\
\hline $\mathrm{T} 1 / \mathrm{T} 2$ & Reference & Reference \\
\hline T3 & $2.15(0.46-10.12)$ & $3.9(0.62-24.42)$ \\
\hline T4 & $0.70(0.12-4.17)$ & $0.76(0.11-5.36)$ \\
\hline \multicolumn{3}{|l|}{ Stage } \\
\hline 1 & Reference & - \\
\hline$\|$ & $0.81(0.08-7.75)$ & - \\
\hline III & $3.67(0.47-29.00)$ & - \\
\hline \multicolumn{3}{|l|}{ Tumor grade } \\
\hline I & Reference & - \\
\hline$\|$ & $0.93(0.12-7.31)$ & - \\
\hline III & $0.81(0.07-9.03)$ & - \\
\hline \multicolumn{3}{|l|}{ Upstage } \\
\hline $\mathrm{NO}(\mathrm{i}+)$ & $10.28(1.44-73.34)^{*}$ & $20.32(2.09-197.98)^{*}$ \\
\hline NO & Reference & Reference \\
\hline $\mathrm{N}+$ & $7.93(1.71-36.83)^{*}$ & $10.06(2.04-49.58)^{*}$ \\
\hline
\end{tabular}

HE examination

$\begin{array}{ll}\text { Negative } & \text { Reference } \\ \text { Positive } & 4.82(1.48-15.70)^{*} \\ \text { SLN examination } & \\ \text { Negative } & \text { Reference } \\ \text { Positive } & 1.50(0.33-6.84)\end{array}$

$\mathrm{HE}$, hematoxylin-eosin; SLN, sentinel lymph node.

Dash denotes variables were not included in the final model.

* Indicates $\mathrm{p}<0.05$.

${ }^{\dagger}$ Ratio values were multiplied by 100 .

(30 of 141) of the patients, classified as pN0 by routine histopathological examination, were found to have micrometastasis or isolated tumor cells in the SLN [20]. Interestingly, a study by Cheng et al. [28] of colorectal cancer patients and liver metastases showed that nuclear beta-catenin overexpression in metastatic lymph nodes was strongly associated with liver metastasis. Perin et al. [29] reported a case a 46-year-old female who underwent sigmoid colon resection for colon cancer who subsequently developed pathologically proven breast metastasis of the colon malignancy. Examination of the 
Table 5 Cox proportional hazard model for recurrence or/ and metastasis

\begin{tabular}{|c|c|c|}
\hline & Univariate & Multiple \\
\hline Age (y) & $0.98(0.95-1.02)$ & $1.00(0.97-1.04)$ \\
\hline Lymph node ratio $^{+}$ & $1.06(1.02-1.11)^{*}$ & - \\
\hline \multicolumn{3}{|l|}{ Gender } \\
\hline Female & Reference & Reference \\
\hline Male & $1.75(0.55-5.58)$ & $2.19(0.59-8.20)$ \\
\hline \multicolumn{3}{|l|}{ Location of tumor } \\
\hline Colon & Reference & - \\
\hline Rectum & $1.16(0.40-3.30)$ & - \\
\hline \multicolumn{3}{|l|}{ Depth of invasion } \\
\hline $\mathrm{T} 1$ or $\mathrm{T} 2$ & Reference & - \\
\hline T3 & $2.23(0.47-10.51)$ & - \\
\hline T4 & $1.03(0.19-5.63)$ & - \\
\hline \multicolumn{3}{|l|}{ Stage } \\
\hline । & Reference & - \\
\hline$\|$ & $0.83(0.09-8.02)$ & - \\
\hline III & $4.60(0.59-36.07)$ & - \\
\hline \multicolumn{3}{|l|}{ Tumor grade } \\
\hline । & Reference & - \\
\hline$\|$ & $0.95(0.12-7.41)$ & - \\
\hline III & $0.69(0.06-7.67)$ & - \\
\hline \multicolumn{3}{|l|}{ Upstage } \\
\hline $\mathrm{NO}(\mathrm{i}+)$ & $9.97(1.40-71.07)^{*}$ & $8.07(1.09-59.50)^{*}$ \\
\hline No & Reference & Reference \\
\hline $\mathrm{N}+$ & $9.67(2.10-44.47)^{*}$ & $7.87(1.68-36.79)^{*}$ \\
\hline \multicolumn{3}{|l|}{ HE examination } \\
\hline Negative & Reference & - \\
\hline Positive & $5.93(1.84-19.12)^{*}$ & - \\
\hline \multicolumn{3}{|l|}{ SLN examination } \\
\hline Negative & Reference & - \\
\hline Positive & $1.11(0.25-4.96)$ & - \\
\hline
\end{tabular}

HE, hematoxylin-eosin; SLN, sentinel lymph node.

Dash denotes variables were not included in the final model.

* Indicates $\mathrm{p}<0.05$.

${ }^{\dagger}$ Ratio values were multiplied by 100 .

axillary SLN revealed metastasis consistent with the colon primary.

Despite the encouraging results, there remains controversy regarding false negative rates. Our false negative rate was $68.2 \%$, higher than that reported by other authors, and our results indicated that the SLN did not predict overall nodal status. This is similar to the findings of a recent systematic review and meta-analysis performed by van der Pas et al. [1]. The authors included 52 studies with 3767 SLN procedures (78.6\% colon carcinoma, $21.4 \%$ rectal carcinoma) and found a mean detection rate of $94 \%$ and a pooled sensitivity of $76 \%$. Retter et al. [30] reported the results of 31 patients who received surgery for colon carcinoma in which in vivo SLN mapping was performed, and found that although the SLN was identified in 28 of the 31 patients, the false-negative rate to identify stage III disease was $66 \%$ and the accuracy was $14 \%$. Finan et al. [31] also reported that ex vivo SLN mapping did not improve staging after proctectomy for rectal cancer. In contrast, van Schaik et al. [4] reported no false negative SLNs in 44 patients. The reasons for the high false-negative rates in some studies are unclear, but may possibly include the T stage, tumor location, and learning curve for SLN mapping. Thus, we believe that the cumulative data indicate that routine SLN detection alone cannot replace the conventional method that examines all dissected lymph nodes because a high false-negative rate tends to downgrade tumor staging such that some patients who may benefit from adjuvant chemotherapy will not receive it.

Although almost all studies analyzing SLN mapping for colorectal cancer demonstrate the detection of micrometastasis, the clinical significance of lymph node micrometastasis, particularly that identified solely by IHC staining, is unknown. There have been extensive studies on lymph node micrometastasis using IHC or RT-PCR techniques, and some reports have indicated that micrometastasis detection by $\mathrm{ICH}$ was associated with poor prognosis of colon cancers [8-10]. However, other studies have reported conflicting findings [5,11]. Dahl et al. [18] reported that only patients with metastatic lymph nodes detected directly or within a SLN died of metastatic disease. Saha et al. [19] demonstrated with a 2-year minimum follow-up of 153 patients who underwent SLN mapping, $7 \%$ had recurrences as compared with $25 \%$ of 162 patients with standard resection and nodal staging. Bilchik et al. [27] reported that no colon cancer patient with a negative SLN by HE and PCR had a recurrence at a mean follow-up of 25 months. Our results indicated that patients without lymph node metastasis by HE staining who are upstaged by ICH detection of micrometastasis have a significantly poorer OS and DFS. However, survival analysis showed no difference in OS and DFS between the stage III and stage NO(i+) groups. This may imply that patients with SLN micrometastasis have the same prognosis as patients with stage III disease, which suggests that focusing on the detection of SLN micrometastasis could become a useful factor in determining prognosis, although further study is warranted due to limited number of cases. In addition, in multivariate analysis upstaging by SLN micrometastasis identification was not an independent prognostic factor; the small number of study patients may be the reason.

The primary limitation of the study is the relatively small number of patients. However, the results do support those of other studies of the utility of SLN mapping and IHC detection of micrometastasis. 


\section{Conclusions}

Our findings indicate that SLN detection is feasible for colorectal cancers, and ex vivo localization and micrometastasis detection of SLNs in colorectal cancers can upgrade tumor staging. Though SNL examination in combination with IHC for the detection of micrometastasis can reduce the false-negative rate, the method is not suggested to replace the conventional $\mathrm{N}$-staging method that examines all dissected lymph nodes. Moreover, ex vivo localization and micrometastasis detection of SLNs in colorectal cancers may become a factor affecting prognosis and guiding treatment.

\section{Competing interests}

The author(s) declare that they have no competing interests.

\section{Authors' contributions}

F-LW, FS have made substantial contributions to conception and design, acquisition of data, analysis of data; involved in drafting the manuscript. DSW, Z-HL, L-RL, GC, X-JW, P-RD, L-HK participated in the design of the study; involved in revising manuscript critically for important intellectual content. ZZP have made substantial contributions to conception and design; involved in revising it critically for important intellectual content. All authors read and approved the final manuscript.

Received: 14 March 2012 Accepted: 29 May 2012

Published: 22 June 2012

\section{References}

1. van der Pas MH, Meijer S, Hoekstra OS, Riphagen II, de Vet HC, Knol DL, van Grieken NC, Meijerink WJ: Sentinel-lymph-node procedure in colon and rectal cancer: a systematic review and meta-analysis. Lancet Oncol 2011, 12:540-550.

2. Cunningham D, Atkin W, Lenz HJ, Lynch HT, Minsky B, Nordlinger B, Starling N: Colorectal cancer. Lancet 2010, 375:1030-1047.

3. Wiese D, Sirop S, Yestrepsky B, Ghanem M, Bassily N, Ng P, Liu W, Quiachon E, Ahsan A, Badin J, Saha S: Ultrastaging of sentinel lymph nodes (SLNs) vs. non-SLNs in colorectal cancer--do we need both? Am J Surg 2010, 199:354-358. discussion 8.

4. van Schaik PM, van der Linden JC, Ernst MF, Gelderman WA, Bosscha K: Ex vivo sentinel lymph node "mapping" in colorectal cancer. Eur J Surg Oncol 2007, 33:1177-1182.

5. Lips DJ, Koebrugge B, Liefers GJ, van de Linden JC, Smit VT, Pruijt HF, Putter $H$, van de Velde $C J$, Bosscha $K$ : The influence of micrometastasis on prognosis and survival in stage I-II colon cancer patients: the Enroute $\oplus$ Study. BMC Surg 2011, 11:11.

6. Baxter NN, Virnig DJ, Rothenberger DA, Morris AM, Jessurun J, Virnig BA Lymph node evaluation in colorectal cancer patients: a populationbased study. J Natl Cancer Inst 2005, 97:219-225.

7. Bilimoria KY, Bentrem DJ, Stewart AK, Talamonti MS, Winchester DP, Russell $\mathrm{TR}$, Ko CY: Lymph node evaluation as a colon cancer quality measure: a national hospital report card. J Natl Cancer Inst 2008, 100:1310-1317.

8. Faerden AE, Sjo OH, Bukholm IR, Andersen SN, Svindland A, Nesbakken A, Bakka A: Lymph node micrometastasis and isolated tumor cells influence survival in stage I and II colon cancer. Dis Colon Rectum 2011, 54:200-206.

9. Wasif $N$, Faries MB, Saha S, Turner RR, Wiese D, McCarter MD, Shen $P$, Stojadinovic A, Bilchik AJ: Predictors of occult nodal metastasis in colon cancer: results from a prospective multicenter trial. Surgery 2010, 147:352-357.

10. Bosch Roig CE, Roselló-Sastre E, Alonso Hernández S, Almenar Cubells D, Grau Cardona E, Camarasa Lillo N, Bautista D, Molins Palau C: Prognostic value of the detection of lymph node micrometastasis in colon cancer. Clin Transl Oncol 2008, 10:572-578.
11. Bilchik AJ, Stojadinovic A, Wainberg Z, Hecht JR: Targeted lymph node evaluation in colorectal cancer: a decade of progress! J Surg Oncol 99, 99:273-274

12. Salhab M, Patani N, Mokbel K: Sentinel lymph node micrometastasis in human breast cancer: an update. Surg Oncol 2011, 20:e195-e206.

13. Osako T, Iwase T, Kimura K, Yamashita K, Horii R, Yanagisawa A, Akiyama F: Intraoperative molecular assay for sentinel lymph node metastases in early stage breast cancer: a comparative analysis between one-step nucleic acid amplification whole node assay and routine frozen section histology. Cancer 2011, 117:4365-4374.

14. Yi M, Giordano SH, Meric-Bernstam F, Mittendorf EA, Kuerer HM, Hwang RF, Bedrosian I, Rourke L, Hunt KK: Trends in and outcomes from sentinel lymph node biopsy (SLNB) alone vs. SLNB with axillary lymph node dissection for node-positive breast cancer patients: experience from the SEER database. Ann Surg Oncol 2010, 17(Suppl 3):343-351.

15. Dragan R, Nebojsa M, Dejan S, Ivan P, Dragos S, Damir J, Predrag S, Vladan Z: Clinical application of sentinel lymph node biopsy for staging, treatment and prognosis of colon and gastric cancer. Hepatogastroenterology 2009, 56:1606-1611.

16. Des Guetz G, Uzzan B, Nicolas P, Cucherat M, de Mestier P, Morere JF, Breau JL, Perret $G$ : Is sentinel lymph node mapping in colorectal cancer a future prognostic factor? A meta-analysis. World J Surg 2007, 31:1304-1312.

17. Bembenek A, String A, Gretschel S, Schlag PM: Technique and clinical consequences of sentinel lymph node biopsy in colorectal cancer. Surg Oncol 2008, 17:183-193.

18. Dahl K, Westlin J, Kraaz W, Winqvist O, Bergkvist L, Thorn M: Identification of sentinel nodes in patients with colon cancer. Eur J Surg Oncol 2005, 31:381-385.

19. Saha S, Sehgal R, Patel M, Doan K, Dan A, Bilchik A, Beutler T, Wiese D, Bassily $N$, Yee C: A multicenter trial of sentinel lymph node mapping in colorectal cancer: prognostic implications for nodal staging and recurrence. Am J Surg 2006, 191:305-310.

20. Bembenek AE, Rosenberg R, Wagler E, Gretschel S, Sendler A, Siewert JR, Nährig J, Witzigmann H, Hauss J, Knorr C, Dimmler A, Gröne J, Buhr HJ, Haier J, Herbst H, Tepel J, Siphos B, Kleespies A, Koenigsrainer A, Stoecklein NH, Horstmann O, Grützmann R, Imdahl A, Svoboda D, Wittekind C, Schneider W, Wernecke KD, Schlag PM: Sentinel lymph node biopsy in colon cancer: a prospective multicenter trial. Ann Surg 2007, 245:858-863.

21. Quadros CA, Lopes A, Araujo I, Fregnani JH, Fahel F: Upstaging benefits and accuracy of sentinel lymph node mapping in colorectal adenocarcinoma nodal staging. J Surg Oncol 2008, 98:324-330

22. Stojadinovic A, Nissan A, Protic M, Adair CF, Prus D, Usaj S, Howard RS, Radovanovic D, Breberina M, Shriver CD, Grinbaum R, Nelson JM, Brown TA, Freund HR, Potter JF, Peretz T, Peoples GE: Prospective randomized study comparing sentinel lymph node evaluation with standard pathologic evaluation for the staging of colon carcinoma: results from the United States Military Cancer Institute Clinical Trials Group Study GI-01. Ann Surg 2007, 245:846-857.

23. van der Zaag ES, Buskens CJ, Kooij N, Akol H, Peters HM, Bouma WH, Bemelman WA: Improving staging accuracy in colon and rectal cancer by sentinel lymph node mapping: a comparative study. Eur J Surg Oncol 2009, 35:1065-1070

24. Bertoglio S, Sandrucci S, Percivale P, Goss M, Gipponi M, Moresco L, Mussa B, Mussa A: Prognostic value of sentinel lymph node biopsy in the pathologic staging of colorectal cancer patients. J Surg Oncol 2004, 85:166-170.

25. Sandrucci S, Mussa B, Goss M, Mistrangelo M, Satolli MA, Sapino A, Bellò M, Bisi G, Mussa A: Lymphoscintigraphic localization of sentinel node in early colorectal cancer: results of a monocentric study. J Surg Oncol 2007, 96:464-469.

26. Wiese D, Saha S, Yestrepsky B, Korant A, Sirop S: A prospective study of false-positive diagnosis of micrometastatic cells in the sentinel lymph nodes in colorectal cancer. Ann Surg Oncol 2009, 16:2166-2169.

27. Bilchik AJ, Hoon DS, Saha S, Turner RR, Wiese D, DiNome M, Koyanagi K, McCarter M, Shen P, Iddings D, Chen SL, Gonzalez M, Elashoff D, Morton DL: Prognostic impact of micrometastasis in colon cancer: interim results of a prospective multicenter trial. Ann Surg 2007, 246:568-575. discussion 75-7.

28. Cheng $H$, Liang $H$, Qin $Y$, Liu Y: Nuclear beta-catenin overexpression in metastatic sentinel lymph node is associated with synchronous liver metastasis in colorectal cancer. Diagn Pathol 2011, 6:109. 
29. Perin $T$, Canzonieri $V$, Memeo L, Massarut $S$ : Breast metastasis of primary colon cancer with micrometastasis in the axillary sentinel node: a metastasis that metastasized? Diagn Pathol 2011, 6:45.

30. Retter SM, Herrmann G, Schiedeck TH: Clinical value of sentinel node mapping in carcinoma of the colon. Colorectal Dis 2011, 13:855-859.

31. Finan KR, Lewis JS Jr, Winslow E, Mutch MG, Birnbaum EH, Fleshman JW: Ex vivo sentinel lymph node mapping in patients undergoing proctectomy for rectal cancer. Dis Colon Rectum 2010, 53:243-250.

doi:10.1186/1746-1596-7-71

Cite this article as: Wang et al:: Ex vivo localization and

immunohistochemical detection of sentinel lymph node

micrometastasis in patients with colorectal cancer can upgrade tumor

staging. Diagnostic Pathology 2012 7:71.

\section{Submit your next manuscript to BioMed Central and take full advantage of:}

- Convenient online submission

- Thorough peer review

- No space constraints or color figure charges

- Immediate publication on acceptance

- Inclusion in PubMed, CAS, Scopus and Google Scholar

- Research which is freely available for redistribution 